\title{
POLYARTHRITIS IN PEDIATRIC INFLAMMATORY BOWEL DISEASE - A CASE REPORT
}

\author{
Ninel REVENC0 ${ }^{1,2} \unrhd$, Lucia MAZUR-NICORICI ${ }^{1}$, Dina BUJOR ${ }^{1,2}$, Ana-Mihaela BALANUTA ${ }^{1,2}$, \\ Silvia FOCA ${ }^{1,2}$, Ion MIHU1,2, Olga GAIDARJI ${ }^{1}$ \\ ${ }^{1}$ State University of Medicine and Pharmacy "Nicolae Testemitanu", Chisinau, Republic of Moldova \\ ${ }^{2}$ Public Healthcare Institution - Mother and Child Institute, Chisinau, Republic of Moldova \\ Received 06 Nov 2020, Accepted 19 Nov 2020 \\ https://doi.org/10.31688/ABMU.2020.55.4.20
}

\begin{abstract}
Introduction. Approaching the pediatric patient with joint pain is often a challenge, even for the most experienced clinicians. The diagnosis of inflammatory bowel disease in children may be difficult because of atypical symptoms and/or extraintestinal manifestations such as arthritis, weight loss, chronic anemia, unexplained fever.

Case presentation. We present the case of a 13-year-old girl, presented initially with musculoskeletal complains, feverish, weight loss. After an extensive clinical and paraclinical examination and a proper differential diagnosis, the diagnosis of inflammatory bowel disease was established, confirmed by immunological investigations, colonoscopy with biopsy. The peculiarity of the clinical case translates into the onset of ulcerative colitis through extraintestinal manifestations, the main clinical manifestations being related to articular involvement.
\end{abstract}

Conclusions. Overlap syndromes are a main challenge for clinicians. Inflammatory polyarticular syndrome in a child can often result in a benign self-limit ing disease, but sometimes may reveal a serious chronic disease with significant morbidity. Ulcerative colitis diagnosed in children presenting with an amalgam of

\section{Résumé}

La polyarthrite dans la maladie inflammatoire de l'intestin chez l'enfant- présentation de cas

Introduction. Le patient pédiatrique souffrant de douleurs articulaires est souvent difficile d'aborder, même pour les cliniciens les plus expérimentés. Le diagnostic d'une maladie inflammatoire de l'intestin peut être difficile d'établir à cause de symptômes atypiques et/ou des manifestations extra-intestinales comme l'arthrite, la perte de poids, l'anémie chronique, la fièvre inexpliquée.

Présentation de cas. C'est le cas d'une jeune fille de 13 ans, qui présentait au début des plaintes musculosquelettiques, de la fièvre, une perte de poids. Après un examen complet et un diagnostic différentiel approprié, on a soupçonné un début de la maladie inflammatoire de l'intestin. Le diagnostic a été confirmé par les investigations immunologiques, la coloscopie avec biopsie. La particularité du cas clinique se traduit par l'apparition d'une colite ulcéreuse aux manifestations extra-intestinales, parmi lesquelles la plus importante était l'implication articulaire.

Conclusions. Les syndromes à recouvrement constituent un défi majeur pour les cliniciens. Le syndrome 
extraintestinal manifestations requires a multidisciplinary approach.

Keywords: inflammatory polyarticular syndrome, inflammatory bowel disease, children, extraintestinal manifestations.

\section{List of abbreviations:}

CBC-complete blood count

EMG -electromyography

pGALS - pediatric Gait, Arms, Legs and Spine; a simple quick musculoskeletal assessment ANA-anti-nuclear antibody

IBD- inflammatory bowel disease

JIA-juvenile idiopathic arthritis

\section{INTRODUCTION}

Approaching the pediatric patient with joint pain is often a challenge even for the most experienced clinicians. A variety of local and systemic, acute and chronic, benign and malignant clinical conditions are associated with musculoskeletal involvement ${ }^{1}$. The inflammatory nature of arthritis may be due to infection or autoimmune mechanisms. The main landmarks are the finding of the pattern of arthritis, the symmetry ofi the involvement, the axial involvement and the association of the systemic involvement ${ }^{1}$.

The main diseases that must be considered for the differential diagnosis in the case of a child with inflammatory polyarthritis and systemic impairment are the systemic form of the juvenile idiopathic arthritis (JIA), systemic lupus erythematosus (SLE), juvenile systemic sclerosis (JSS), and inflammatory bowel disease (IBD) ${ }^{1}$. The complexity and heterogeneity, as defining features of autoimmune diseases, often translate similarities and overlap syndromes ${ }^{1}$.

IBD is characterized by chronic, recurrent, intermittent inflammation, genetic susceptibility and autoimmune mechanisms being the main pathophysiological factors ${ }^{2}$. About $20 \%$ of all IBDs occur in childhood or adolescence ${ }^{3}$. Ulcerative colitis is a chronic systemic disease, and extraintestinal manifestations are proofs that the inflammatory mechanism is not limited to the intestinal segment ${ }^{4,5}$. The positive diagnosis in children and adolescents may be difficult, due to atypical symptoms and/or extraintestinal manifestations such as arthritis, weight loss, chronic anemia, unexplained fever, mouth ulcers. Musculoskeletal disorders are the most common extraintestinal manifestations in patients with IBD. Joint symptoms occur in up to $40 \%$ of patients with $\mathrm{IBD}^{6}$. They can be divided into peripheral and axial polyarticulaire inflammatoire chez un enfant peut souvent entraîner une maladie bénigne spontanément résolue, mais peut parfois révéler une maladie chronique grave à une morbidité importante. La colite ulcéreuse diagnostiquée chez les enfants présentant un amalgame de manifestations extra-intestinales nécessite une approche multidisciplinaire.

Mots-clés: syndrome polyarticulaire inflammatoire, maladie inflammatoire de l'intestin, enfants, manifestations extra-intestinales.

arthropathy; the peripheral ones are further divided into type 1 and type $2^{6}$. Peripheral arthritis type 1 is an asymmetric oligo arthritis, is more common and usually associated with IBD eruptions. In contrast, peripheral arthritis type 2, presenting as progressive symmetrical polyarthritis, is independent of inflammation of the intestine ${ }^{6,7}$.

Joint manifestations at onset often anticipate the evolution of intestinal syndromes and a complete, targeted and detailed clinical examination is a decisive step for the correct diagnostic orientation. The early diagnosis is essential for prognosis ${ }^{1}$. In the context of clinical polymorphism and often chameleon-like onset, major problems of differential diagnosis are emerging, especially for clinicians who offer initial counseling to the pediatric patient.

\section{Case presentation}

We present the case of a 13-year-old female patient, admitted in our clinic for symmetrical joint swelling, pain and tenderness, mainly in the small joints of the hand and foot, wrist and ankles, with a migratory character. The patient has signed an informed consent for publication of her medical data. She complains also about changes and deterioration in handwriting and other fine motor skills such as getting dressed, and hair brushing. Other complains were: weight loss about $6-8 \mathrm{~kg}$ in $4-5$ weeks, episodic low-grade fever, myalgia, and constipation. According to the heredo-colateral history, maternal grandmother was diagnosed with colon cancer and died. The patient is considered ill for one month, at the beginning left knee was swollen and painful, then the joint involvement developed and gradually appeared symmetrical arthritis and tendinitis. The clinical examination and pediatric Gait, Arms, Legs and Spine (pGALS) at the moment of admission revealed 
palmary edema on the left hand, and tendinitis in the projection of the fourth finger - thickening of the flexor tendon, palpable and painful (Figure 1). Also, we found limited movements in the small joints of the hand (incomplete fist). Decreased muscle strength was appreciated 4/5. No clinical data on impaired internal organs have been identified.

The laboratory tests revealed normal complete blood count (CBC) and C-reactive protein, cytolytic and cholestatic syndromes, increased erythrocyte sedimentation rate (ESR).

Immunological investigations revealed the presence of anti-centromere CNP B antibody and positive anti-DFS70 antibodies. In September 2019, the European League Against Rheumatism (EULAR) and the American College of Rheumatology (ACR) published new criteria for classifying $\mathrm{SLE}^{8}$. The EULAR/ACR criteria have a sensitivity of $96.1 \%$ and a specificity of $93.4 \%$ for the diagnosis. EULAR/ ACR classification requires an antinuclear antibody titer (ANA) of at least 1:80 on HEp-2 cells followed by additive weighted criteria grouped in 7 clinical (constitutional, hematologic, neuropsychiatric, mucocutaneous, serosal, musculoskeletal, renal) and 3 immunologic domains. Each criterion is assigned points, ranging from 2 to 10 . Patients with at least one clinical criterion and 10 or more points are classified as having $\mathrm{SLE}^{8}$. Our patient registered only 8 points, being ineligible for the diagnosis of SLE. International League Against Rheumatism (ILAR), proposed the following criteria for juvenile idiopathic arthritis, systemic form: fever of unknown origin (except infectious, neoplastic, autoimmune or monogenic autoinflammatory diseases) which is documented to be daily (daily; fever increasing to $\geq 39^{\circ} \mathrm{C}$ once a day and returning to $\leq 37^{\circ} \mathrm{C}$ between fever peaks) for at least 3 consecutive days and recurrence for at least 2 weeks and accompanied by 2 major or one major and 2 minor criteria. The major criteria are: evanescent (non-fixed) erythematous rash, and arthritis. Minor criteria: generalized enlargement of the lymph nodes and/ or hepatomegaly and/ or splenomegaly; serosity; arthralgia lasting 2 weeks or more (in the absence of arthritis); and leukocytosis $\left(\geq 15,000 / \mathrm{mm}^{3}\right)$ with neutrophilia.

In the case of our patient, insufficient criteria were found. Immunological testing elucidated controversial results, anti-CENP B antibodies are serological markers for limiting systemic scleroderma previously known as CREST syndrome, but they may be present also in patients with primary biliary cholangitis. The assumption of the solitary diagnosis of primitive biliary cholangitis does not explain all signs and symptoms, especially the joint involvement. At the same time, the presence of anti-DFS70 antibodies has a low significance in systemic autoimmune rheumatic diseases. Therefore, we raised the suspicion of primitive biliary cholangitis and we started a differential diagnosis with limiting systemic scleroderma. According to the EULAR recommendations, with reference to the diagnostic criteria for systemic scleroderma, no sufficient criteria were found. We performed capillaroscopy (Figure 2) and did not find a capillaroscopic scleroderma pattern, the morphology was normal: staple appearance, normal density, 8 capillaries per $\mathrm{mm}$, no bleeding, the architecture- the capillaries are parallel.

According to the recommendations of the European Association for the Study of the Liver (EASL), primitive biliary cholangitis requires a differential diagnosis with autoimmune hepatitis. For exclusion/ confirmation, it is necessary to perform

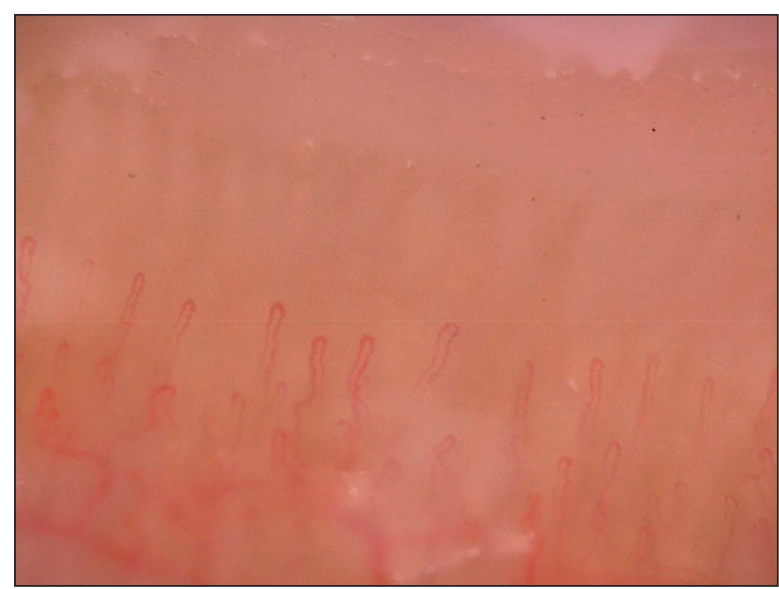

Figure 2. Periungual capillaroscopy. Normal capillaroscopic pattern in a 13 -year-old patient.

Rodnan's skin score did not show skin induration. 


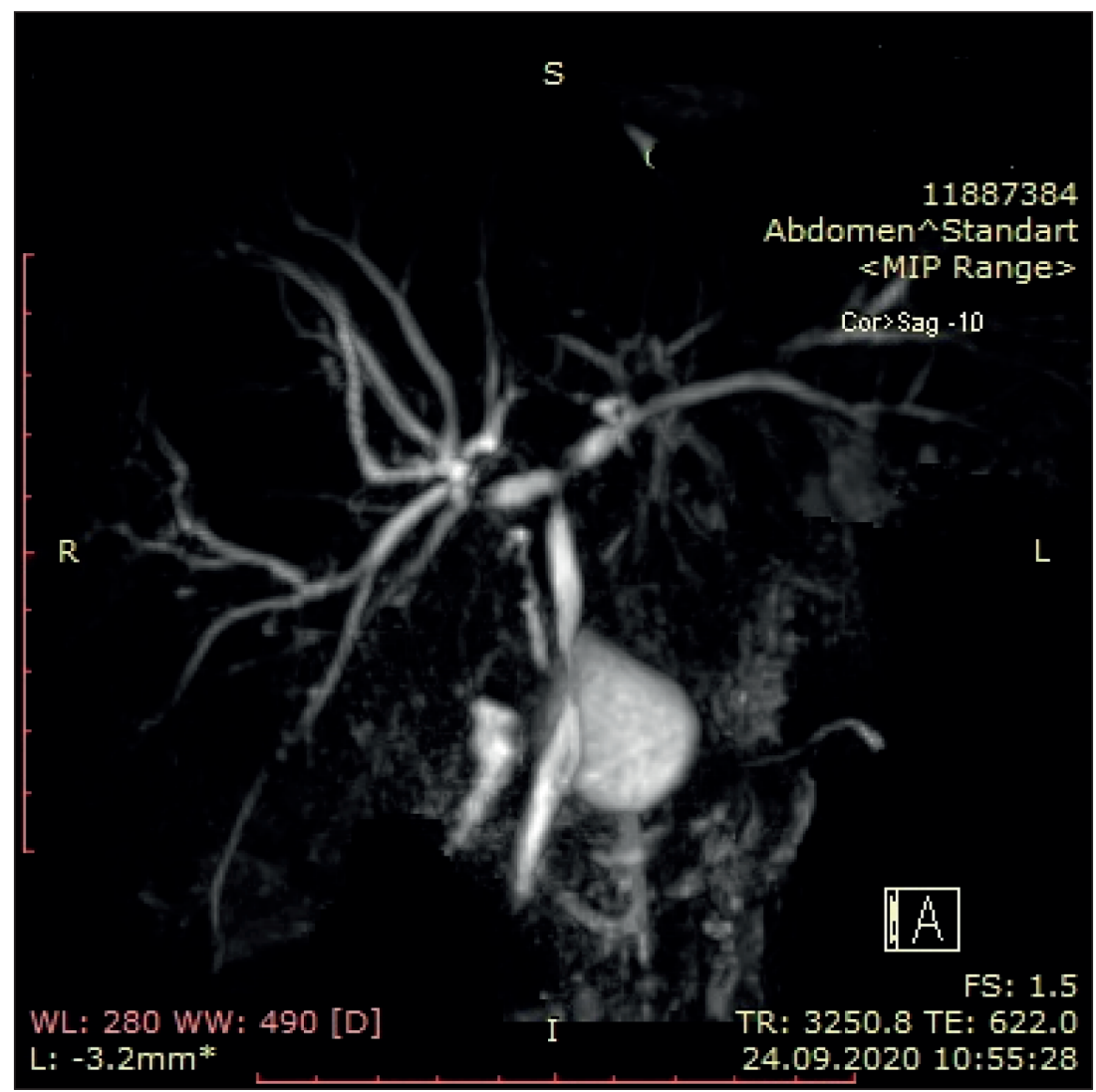

Figure 3. MRI cholangiopancreatography - intra and extrahepatic bile ducts are dilated, with segmental stenosis, without pre-stenotic dilatations. Extra and intrahepatic bile filling defects are not visualized.

immunological investigations aimed at hepatobiliary autoimmune damage and magnetic resonance imaging (MRI) in cholangiopancreatographic regimen (Figure 3). The results (AMA M2, M2-3E, LKM-1, LC-1, SLA/ LP - negative) were not conclusive for any of the diseases.

MRI cholangiopancreatography revealed the presence of segmental stenosis of the intra- and extrahepatic bile ducts, specifying the presence of sclerosing cholangitis (Figure 3). Hepatosplenomegaly was also described. This latest imaging investigation outlined a preliminary clinical diagnosis, which integrates the entire symptomatic, clinical and paraclinical spectrum. Thus, taking into account the extraintestinal manifestations (arthralgias, tendinitis, myalgia, fatigue, weight loss translated by malabsorption syndrome), intestinal manifestations (frequent constipation, blood in the stool - the girl was ashamed to tell her parents and medical workers about this clinical sign elucidated during hospitalization), abnormal biochemical tests (cytolytic syndrome, cholestatic syndrome due to sclerosing cholangitis, elevated ESR), hepatomegaly, sclerosing cholangitis confirmed by MRI, splenomegaly (an enlarged spleen may be indicative of portal hypertension), an IBD was suspected. Primary sclerosing cholangitis is found in up to $90 \%$ of cases in children with IBD- ulcerative colitis ${ }^{8}$. We performed diagnostic colonoscopy with biopsy, to confirm the diagnosis. At the same time, blood samples were taken to make the differential diagnosis between Crohn's disease and ulcerative colitis. Antibodies Saccharomyces cerevisiae (ASCA) IgA/G, were-negative, according to literature data, they are positive in $60 \%-70 \%$ of patients with Crohn's disease ${ }^{9}$. Instead, the positive p-ANCA result (weakly positive in the case of our patient) indicates ulcerative colitis.

Colonoscopy described indirect signs of ulcerative and hemorrhagic colitis (mucosa was moderately hyperemic in the rectum and sigmoid and bleeds to instrumental palpation). Fecal samples were collected for the qualitative assessment of calprotectin, with negative result. The microscopic examination showed: colonic epithelium with marked plasma cell infiltration and eosinophilic inflammatory infiltration.

Treatment with systemic corticosteroids was initiated - methylprednisolone $36 \mathrm{mg}$, divided into 3 doses, gastroprotection, probiotics. The child had a good response to corticosteroid therapy, with amelioration of the joint manifestations. The biochemical tests and ESR returned to normal. At discharge, we recommended the continuation of oral steroids 
for approximately one month, with progressive withdrawal after two weeks, associated with proton pump inhibitors, calcium supplements, and probiotics for one month.

\section{Discussion}

Imaging confirmation of sclerosing cholangitis was decisive for reconsidering the diagnostic algorithm in the case of this patient. Primary sclerosing cholangitis is a chronic cholestatic disease, which is frequently progressive and can lead to cirrhosis ${ }^{11,12}$. The disease is characterized by progressive inflammation, fibrosis and tightening of the intrahepatic and extrahepatic bile ducts, a distinctive moment compared to the primary biliary cholangitis that was initially suspected in this patient. The prevalence of primary sclerosing cholangitis in patients with ulcerative colitis is $90 \%{ }^{12,13}$. Interestingly, the diagnosis of primary sclerosing cholangitis appears to influence the evolution of IBD, because patients with primary sclerosing cholangitis and ulcerative colitis have a milder course of colitis, with less pronounced histological inflammation of the colon. However, the presence of primary sclerosing cholangitis is an independent risk factor for the development of colorectal dysplasia and/or cancer in patients with IBD, leading to the recommendation of annual surveillance colonoscopies in patients with IBD $^{13,14}$.

The peculiarity of the clinical case translates into the onset of ulcerative colitis through extraintestinal manifestations, the main complains referring to joint involvement. Up to $20 \%$ of pediatric patients report arthralgia before gastrointestinal symptoms $s^{6}$. Diagnostic colonoscopy accompanied by biopsy is necessary for the diagnosis, a distinctive sign is the lack of neutrophilic infiltrate, which confirms the presence of a low degree of inflammation in the colon. In other words, eosinophilic infiltrate plays a controversial role ${ }^{16}$. Clinical studies specify that eosinophilic infiltrate may play a role in explaining the different phenotypes of IBD and the variable response to different treatments and the variation in disease severity ${ }^{15}$. Boyle et al, in a recent study targeting the pediatric population, reported that the increased number of colonic eosinophils in children was associated with less severe inflammation on endoscopic examination ${ }^{16}$. In contrast, other studies have shown that tissue eosinophils are associated with a more severe course of the disease $\mathrm{e}^{17}$.

\section{Conclusions}

Overlap syndromes are a main challenge for clinicians. Inflammatory polyarticular syndrome in a child can often result in a benign self-limiting disease, but sometimes may reveal a serious chronic disease with significant morbidity. Ulcerative colitis diagnosed in children presenting with an amalgam of extraintestinal manifestations requires a multidisciplinary approach.

\section{Author Contributions:}

O.G., and I.M. were responsible for the diagnostic procedures, N.R., F.S were responsible for clinical diagnosis, and treatment decisions. N.R., B.D., L.M and A-M.B. wrote the manuscript. All authors have read and agreed to the published version of the manuscript.

\section{Compliance with Ethics Requirements:}

„The authors declare no conflict of interest regarding this article"

„The authors declare that all the procedures and experiments of this study respect the ethical standards in the Helsinki Declaration of 1975, as revised in 2008(5), as well as the national law. Informed consent was obtained from the patient included in the study"

"No funding for this study"

\section{References}

1. Balan S. Approach to joint pain in children. Indian Journal of Pediatrics. 2016;83 (2):135-139.

2. Wine E, El-Matary W, Critch J, et al. Pediatric inflammatory bowel diseases: looking to the future. Frontiers in Pediatrics. 2020;8:56

3. Moon JS. Clinical aspects and treatments for pediatric inflammatory bowel diseases. Pediatric Gastroenterology, Hepatology $\mathbb{E}$ Nutrition. 2019;22:50-56.

4. Cuffari C. Diagnostic considerations in pediatric inflammatory bowel disease management. Gastroenterology $\mathfrak{E}$ Hepatology. 2019;5:775-783.

5. Feuerstein JD, Isaacs KL, Schneider Y, et al; Institute Clinical Guidelines Committee. AGA Clinical Practice Guidelines on the Management of Moderate to Severe Ulcerative Colitis. Gastroenterology. 2020;158(5):1450-1461.

6. Hiller A, Biedermann L, Fournier N, et al. The appearance of joint manifestations in the Swiss inflammatory bowel disease cohort. PLOS ONE. 2019;14(4): e0211554.

7. Levine JS, Burakoff R. Extraintestinal manifestations of inflammatory bowel disease. Gastroenterology $\mathbb{E}$ Hepatology. 2011;7(4):235-41.

8. Kowdley KV. Primary sclerosing cholangitis: Epidemiology and pathogenesis. UpToDate. 2020;1-12.

9. Keiichi M, Mikio N, Hidetoshi T, et al. Antibody markers in the diagnosis of inflammatory bowel disease. World Journal of Gastroenterology. 2016;22(3):1304-10.

10. Aringer M, Costenbader K, Daikh D, et al. European League Against Rheumatism/American College of Rheumatology classification criteria for systemic lupus erythematosus. Ann Rheum Dis. 2019;78 (9):1151-1159.

11. Wilson DC, Russell RK. Overview of pediatric IBD. Seminars in Pediatric Surgery. 2017;26(6):344-348. 
12. Lunder AK, RoksundHov J, Borthne A, et al. Prevalence of sclerosing cholangitis detected by magnetic resonance cholangiography in patients with long-term inflammatory bowel disease. Gastroenterology. 2016;151(4):660-669.e4.

13. Chapman MH, Thorburn D, Hirschfield GM, et al. British Society of Gastroenterology and UK-PSC guidelines for the diagnosis and management of primary sclerosing cholangitis. Gut. 2019;68:1356-1378.

14. Rosen MJ, Dhawan A, Saeed SA. Inflammatory bowel disease in children and adolescents. JAMA Pediatrics. 2015;169(11):1053-60.
15. Canavese G, Villanacci V, Antonelli E, et al. Eosinophilia associated basal plasmacytosis: an early and sensitive histologic feature of inflammatory bowel disease. APMIS.2017;125(3).

16. Boyle B, Collins MH, Wang Z, et al. Histologic correlates of clinical and endoscopic severity in children newly diagnosed with ulcerative colitis. Am J Surg Pathol. 2017;41:1491-8.

17. Alhmoud T, Gremida A, Steele DC, et al. Outcomes of inflammatory bowel disease in patients with eosinophil-predominant colonic inflammation. BMJ Open Gastroenterology. 2020;7(1):e000373 